\title{
Head Mouse
}

\author{
Sagar More. ${ }^{1}$, Datta Kale. ${ }^{2}$, Gauri Phatke ${ }^{3}$, Prof. S.B. Bhosale ${ }^{4}$ \\ UG Scholar, Department of Computer Engineering, JCOE, UOP, Maharashtra ${ }^{1,2,3}$ \\ Professor, Department of Computer Engineering, JCOE, UOP, Maharashtra ${ }^{4}$
}

\begin{abstract}
Head tracking driven virtual computer mouse system called "head mouse", is developed for manipulating hand free perceptual user interfaces. It consists the characteristics of a Robust, Real-time head tracker, Head pose/Motion estimator, Virtual mouse control module. For detecting faces in color images we use the Ad boost algorithm. At the first stage, skin color model in the $\mathrm{YCbCr}$ chrominance space is built to segment pixels which are non-skin-color from the image. Then in the later stages, to remove the noisy regions and fill holes in the skin-color region, the mathematical morphological operators are used that can extract candidate human face regions.

By using this, the cursor position is navigated and fine-tuned by using head tilt or yaw rotation the relative position of tracking window in image space is beneficial under the circumstances of user jumping, extreme movement, large degree rotation, turning around, hand/object occlusion, part face out of camera shooting region and multi-user occlusion. It encourages the applications of Interactive computer games, Machine guidance, Robot control and machine access for disabilities and elders.
\end{abstract}

Keywords: HCI, Perceptual user interfaces, Face tracking, feature detection, cursor control, video processing.

\section{INTRODUCTION}

Today's world provides tremendous opportunities to but there was a vast difference in the process of video improve the human life in well manner to tackle the stream and the methods used for feature detection problem which is associated with the existing system. We however, in camera based eye tracking system the develop an alternative solution that is the head mouse, in implementation differs in the methods used for mapping this we retrieved the human motion parameter in video eye movement to the mouse movement, the wait to click captured at real time by camera. The cursor movement of method was mostly used in implementing the mouse click system can be captured by the motion parameter instead of control.The visual face tracking technique is based on a another human body parts the real-time capture video will 3D model which is driven by a novel camera mouse. A be captured by the web camera.At the $1^{\text {st }}$ stage, skin color camera which is connected to a computer captures human model in the $\mathrm{YCbCr}$ chrominance space is built to segment motion parameters in video at real-time and these motion pixel which are non skin -color from the image then in parameters controls the mouse cursor. Motion parameters later stages to remove the noisy regions and fill holes in from the video are retrieved by visual tracking module and the skin-color region, the mathematical morphological the rule of control is specified by the mouse control operation are used. By this way, we can extract candidate module. This paper mainly focused on a face because the human face regions. The 8- bit color image is converted face is a part which can be used for visual human tracking into gray scale image. After that the image segmentation since the color and shape more precisely studied from the \& feature extraction will be done with the help of the last face appearance allowing system to detect and track with stage,the action of mouse control will be performed.

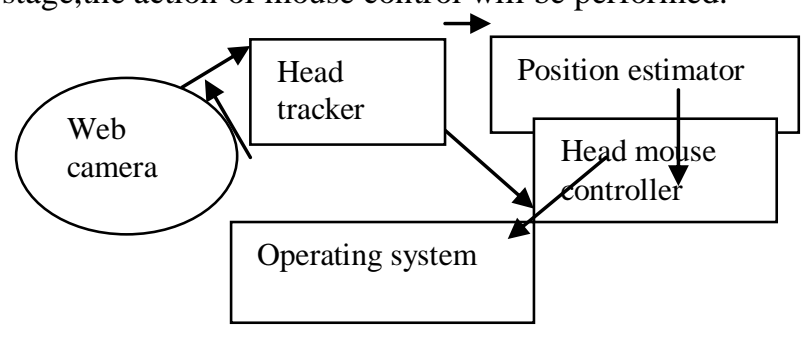

Fig.1 framework of head mouse

\section{LITERATURE SURVEY}

In previous years, the eyes movements tracking was considered as popular method for providing computer control. In this, the camera was connected to computer to track user's eyes. It's designwas same as that of the face detection on the input video stream. It consists the detection of eye location then determining eye movement $\&$ then translating detected movement to mouse control accuracy. Nose 'Use as a mouse', in this paper a nose feature is used for describing face tracking technique. In this system, the user's face position is tracked in a video camera in 3D manner which convert it to a position of cursor ultimately providing hands-free alternative to mouse or trackball. The point on the nose surface which is closer to the camera is considered as a nose feature.

\section{RELATED WORK}

\section{A.HCI}

The main objective is to develop a Ipointing device controlled by head movement that provides the same functionalities as a conventional mouse and it evaluates the performance of the proposed system when it would be operated by quadriplegic users.HCI provides the newer technologies for the people with physical, cognitive, sensory or communicative disabilities related to the field of computer access device and to the field of assistive technology. 


\section{B.PUI}

A PUI allowed the computer users to interact with the computer instead of having to use the normal keyboard and mouse. Those interface realized by giving the computer capability of interpreting the user's movements or voice commands and the hands-free interfacing. Here, we are concerned using the head orientation to control the cursor movement, blinks to simulate mouse clicks and gesture input for the people with physical disabilities, computer from an essential tool for communication, environmental control, education and entertainment.

\section{C.Blur}

In image terms blurring means that each pixel in the source image gets spread over and mixed into surrounding pixels. Blurring an image reduces the sharpening effect; this makes the detection more accurate.

There are two type of blurring an image

- Greyscale blur

- Color blur

Steps for blurring process:

- Traverse through entire input image array.

- Read individual pixel color value (24-bit).

- Split the color value into individual R, G and B 8-bit values.

- Calculate the RGB average of surrounding pixels and assign this average value to it.

- Repeat the above step for each pixel.

- Store the new value at same location in output image.

\section{D.Face Detection:}

Face detection is done by Adaboost algorithm. Using the framework of viola \& Jones the face detection problems are carried out. In this, weak classifiers are considered. A set of Haar-like features are used as the input feature to the cascaded classifiers. In cascading the background regions of the image to be quickly discarded and spending more time on face like features.

\section{E.Face Tracking:}

We are using Mean Shift algorithm for Head pose/motion estimation. For this we required to draw histogram based methods of producing object probability distribution.

In our system track the mode of an objects probability distribution within a video scene. But the probability of distribution of object can change and move dynamically in time, so the mean shift algorithm is modified to deal with dynamically changing probability distribution.

The modified algorithm is called the Continuous Adaptive Mean Shift (CAMSHIFT)[9].

Calculation of mean shift

1. Window size is chosen or search.

2. Choose the initial location in the search window.

3. Compute the mean location.

4. Centre the search window at that location.

5. Repeat step 3 and 4 until convergence are not get.

F.Blob detection:

In the area of computer vision, 'blob detection' refers to visual modules that are aimed at detecting points and/or regions in the image that are either brighter or darker than the surrounding. There are two main classes of blob detectors (i) differential methods based on derivative expressions and (ii) methods based on local extrema in the intensity landscape. With the more recent terminology used in the field, these operators can also be referred to as interest point operators, or alternatively interest region operators. There are several motivations for studying and developing blob detectors. One main reason is to provide complementary information about regions, which is not obtained from edge detectors or corner detectors. In early work in the area, blob detection was used to obtain regions of interest for further processing. These regions could signal the presence of objects or parts of objects in the image domain with application to object recognition and/or object tracking.

G.RGB and $H S V$ :

Color vision can be processed using RGB color space or HSV color space. RGB color space describes colors in terms of the amount of red, green, and blue present. HSV color space describes colors in terms of the Hue, Saturation, and Value. In situations where color description plays an integral role, the HSV color model is often preferred over the RGB model. The HSV model describes colors similarly to how the human eye tends to perceive color. RGB defines color in terms of a combination of primary colors, whereas, HSV describes color using morefamiliar comparisons such as color, vibrancy and brightness. The basketball robot uses HSV color space to process color vision.

- Hue represents the color type. It can be described in terms of an angle on the abovecircle. Although a circle contains 360 degrees of rotation, the hue value is normalized toa range from 0 to 255 , with 0 being red.

- Saturation represents the vibrancy of the color. Its value ranges from 0 to 255 . Thelower the saturation value, the more gray is present in the color, causing it to appear faded.

- Value represents the brightness of the color. It ranges from 0 to 255 , with 0 beingcompletely dark and 255 being fully bright.

H.Cursor Control:

Controlling the cursor position of a PC will be done by the head mouse system. The system provides a free program that allows us to control the pointer of mouse which will be moving by our head.

I.Video Processing:

Firstly the video will be captured by a web camera at realtime to extract the human motion parameters. Then after that the information will be derived which we required from video data captured.

\section{ARCHITECTURE}

\section{A.Web Cam Interfacing:}

Web cam interfacing will be used to provide the better interfacing mechanism in image processing technologies. The real-time video will be captured by the web camera.

B.Frame Grab:-

Frame grab permits the user to capture, maintain or record higher resolution and the increased frame rate of VGA display signal. Frame grab will be more useful to record the video from a computerized device to create a training material like an ultrasound machine. 


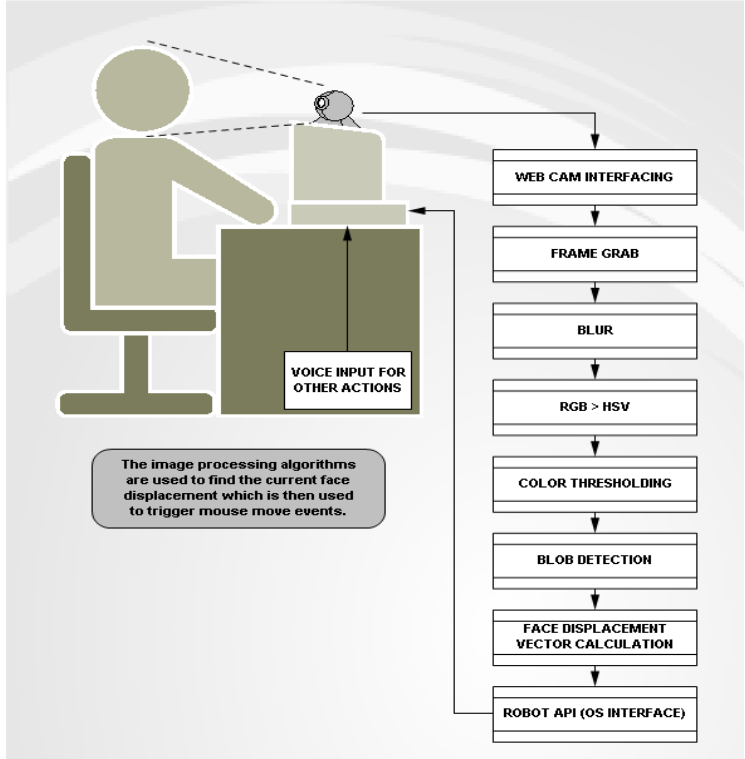

Fig.2 Architecture of head mouse

C.Blur: -

In image terms blurring means that each pixel in the source image gets spread over and mixed into surrounding pixels. Blurring an image reduces the sharpening effect; this makes the detection more accurate. We are doing blur by calculating the average of surrounding 8 pixels that is $3 * 3$ windows. To increase the blur effect, we can scan surrounding 5 pixels that is $5 * 5$ windows.

D.RGB >HSV: -

RGB color space describes colors in terms of the amount of Red, Green, and Blue and HSV color space describes colors in terms of the Hue, Saturation, and Value. The HSV model describes colors similarly to how the human eye tends to perceive color, that's why we are converting RGB values into HSV.

\section{E.Blob detection: -}

'blob detection' refers to visual modules that are aimed at detecting points and/or regions in the image that are either brighter or darker than the surrounding. Blob detection was used to obtain regions of interest for further processing. These regions could signal the presence of objects or parts of objects in the image domain with application to object recognition and/or object tracking.

F.Color Thresholding:Thresholding the simplest method of image segmentation. From a greyscale image, thresholding can be used to create binary images.

\section{G.Face Detection Vector Calculation: -}

At the time of face detection stage, the instantaneous location of the face is compared with their at rest location and then calculates the face displacement vector. The face displacement determines the magnitude and direction of the cursor movement (to do the next face detection).

\section{H. Robot API: -}

This class is used to generate native system input events for the purposes of test automation, self-running demos, and other applications where control of the mouse and keyboard is needed.

\section{CONCLUSION}

Head Mouse has the ability to replace all keyboard and mouse functions that are successfully implemented. User successfully performs the actions like do left click and right click events, drag and drop events etc. It provides the rapid and robust performance in real world applications. System provides user friendly interaction with the computer as well as powerful and more efficient control of a computer.Head Mouse is an alternative solution for future portable devices like PDA, GPS, Cell Phone.

It will be useful in interactive computer games, robot control and mainly accessing the computer for the elder and disabled people.

\section{ACKNOWLEDGMENT}

We would like to express our gratitude to all those who helped us to complete this work. We want to thank our guide Prof. S.B Bhosale for his continuous help and generous assistance. He helped in a broad range of issues from giving us direction, helping to find the solutions, outlining the requirements and always having the time to see us.

We have furthermore to thank Prof. D. N. Wavhal, Head of the Department of Computer Engineering to encourage us to go ahead and for continuous guidance. We also want to thank to all staff for all their assistance and guidance for preparing paper.

We would like to thank our colleagues who helped us time to time from preparing paper and giving good suggestions. We also extend sincere thanks to all the staff members of Department of Computer Engineering for helping us in various aspects.

\section{REFERENCES}

[1] J.L. Tu, T. Huang, and H. Tao, "Face as Mouse through Visual Face Tracking," CVIU special issue on V4HCI, 2006.

[2] J.L. Tu, T. Huang, and H. Tao, "Face as Mouse through Visual Face Tracking," IEEE CRV'05, pp. 339-346, 2005.

[3] T. Morris, and V. Chauhan, "Facial Feature Tracking for Cursor Control," Journal of Network and Computer Applications, Vol.29, No.1, pp. 62-80, 2006.

[4] Dmitry O. Gorodnichy, Shahzad Malik, and Gerhard Roth, "Nouse 'Use Your Nose as a Mouse' - a New Technology for Hands-free Games and Interfaces," VI'02, pp. 354-360, 2002.

[5] T. Yang, S.Z. Li, Q. Pan, J. Li, and C.H. Zhao, "Reliable and Fast Tracking of Faces under Varying Pose," IEEE FGR'06, pp: 421$428,2006$.

[6] K. Toyama, “'Look, Ma - no hands!' Hands-free Cursor Controlwith Real-time 3D FaceTracking," Proc. Workshop on Perceptual User Interfaces (PUI'98), San Fransisco, 1998

[7] M. La Cascia, S. Sclaroff, and V. Athitsos, "Fast, Reliable Head Tracking Under Varying Illumination: An Approach Based on Registration of Texture-Mapped 3D Models," IEEETrans. on PAMI, Vol. 22, No. 4, pp. 322-336, 2000.

[8] H. Tao, and Thomas S. Huang, "Explanation-Based Facial Motion Tracking Using a Piecewise B'ezier Volume Deformation Model," IEEE CVPR'99, Vol. 1, pp. 611-617, 1999.

[9] H. Nanda, and K. Fujimura, "A Robust Elliptical Head Tracker," IEEE FGR'04, pp. 469-474, 2004

[10]G.R. Bradski, "Real Time Face and Object Tracking as a Component of a Perceptual User Interface," IEEE Workshopon WACV '98, pp. 214-219, 1998 